\title{
Influence of Gender, Power, and Hand Position on Pelvic Motion during Seated Cycling
}

\author{
JULIE L. SAUER ${ }^{1}$, JAMES J. POTTER ${ }^{1}$, CHRISTINE L. WEISSHAAR ${ }^{1}$, HEIDI-LYNN PLOEG ${ }^{1,2}$, \\ and DARRYL G. THELEN ${ }^{1-3}$ \\ Departments of ${ }^{1}$ Biomedical Engineering, ${ }^{2}$ Mechanical Engineering, and ${ }^{3}$ Orthopedics and Rehabilitation, \\ University of Wisconsin-Madison, Madison, WI
}

\begin{abstract}
SAUER, J. L., J. J. POTTER, C. L. WEISSHAAR, H.-L. PLOEG, D. G. THELEN. Influence of Gender, Power, and Hand Position on Pelvic Motion during Seated Cycling. Med. Sci. Sports Exerc., Vol. 39, No. 12, pp. 2204-2211, 2007. Introduction/Purpose: An understanding of normal pelvic motion during seated cycling is relevant to saddle design and bicycle fitting. In this study, we investigated the effects of gender, power, and hand position on pelvic motion throughout a pedal stroke. We also investigated whether anthropometric factors could explain any interindividual differences observed. Methods: Twelve experienced male and 14 experienced female cyclists participated. Each subject was custom fitted to a stationary bicycle and then rode the bicycle at three power outputs (100, 150 , and $200 \mathrm{~W}$ ), with their hands in the tops and drops position. The kinematics of a triad of motion-capture markers, located on posterior pelvic landmarks, were used to characterize pelvic motion. Results: The largest angular excursions were observed in the nonsagittal planes, with the pelvis rotating internally $\left(\sim 3^{\circ}\right)$ and rolling laterally $\left(\sim 2^{\circ}\right)$ toward the downstroke. These pelvic rotations caused the hip on the downstroke side to translate anteriorly and inferiorly. Compared with males, females exhibited greater average anterior pelvic tilt in the drops hand position (males: $21 \pm 3^{\circ}$; females: $24 \pm 4^{\circ} ; P=0.036$ ). Interindividual differences in pelvic motion could not be independently explained by measures of ischial tuberosity width or hamstring flexibility. However, average anterior pelvic tilt was negatively correlated with lumbar flexibility among the males $(r=0.75 ; P=0.024)$, suggesting that this may be an important factor to consider in bicycle fitting. Conclusions: We observed substantial pelvic motion during seated cycling, with experienced female road cyclists exhibiting greater average anterior tilt than their male counterparts. Pelvic motion seems to arise naturally during seated cycling and should be considered when designing saddles and establishing bicycle-fitting procedures. Key Words: MOTION CAPTURE, KINEMATICS, HIP MOTION, SADDLE, BICYCLE FITTING
\end{abstract}

$\mathrm{P}$ elvic motion during cycling is often associated with improper bicycle fit and saddle-related pathologies. For example, excessive pelvic roll (i.e., side-to-side rocking motion) is considered indicative of too high a saddle position (12). In addition, chafing has been attributed to the repeated sliding of the soft tissue across the saddle $(9,18,19)$. However, some pelvic motion may be natural and

Address for correspondence: Heidi Ploeg, Ph.D., Department of Mechanical Engineering, 1513 University Ave., Madison, WI 53706; E-mail: ploeg@engr.wisc.edu.

Submitted for publication December 2006.

Accepted for publication July 2007.

0195-9131/07/3912-2204/0

MEDICINE \& SCIENCE IN SPORTS \& EXERCISE E $_{\circledast}$ Copyright (C) 2007 by the American College of Sports Medicine

DOI: $10.1249 / \mathrm{mss} .0 \mathrm{~b} 013 \mathrm{e} 3181568 \mathrm{~b} 66$ facilitate the transfer of energy from the upper to lower body via the hip joint reaction force $(6,14,22)$.

In many prior studies, pelvic motion either has not been directly measured $(2,10,18,20)$ or the pelvis has been assumed to be stationary on the saddle $(5,15,20,21)$. Others have used a single marker located over the greater trochanter to estimate hip motion $(7,22)$. Neptune and Hull (14), using bone pins inserted into the pelvis, have shown that a single-marker approach overestimates hip motion. However, hip motion was still substantial enough for the hip joint reaction force to transfer a considerable amount of energy at higher power outputs. Because of the severity of the bone pin procedure, only a single subject was tested.

Further investigation of normal pelvic motion is important to quantify biomechanical aspects of saddle design and bicycle fit. In particular, the influence of gender is important to consider, given that inherent differences in pelvic anthropometry may influence position and orientation. In addition, 
an increase in power during cycling may be accompanied by a change in pelvic motion (14). Furthermore, joint flexibility and hand position may influence pelvic tilt, which can then lead to a change in kinematics and loading on the saddle (3). The purpose of the current study was to investigate the effects of gender, power, and hand position on three-dimensional pelvic motion during seated stationary cycling. We hypothesized that pelvic tilt would increase with a change of hand position from the tops to the drops, and that pelvic motion would increase with power. We also assessed whether intersubject differences in pelvic motion could be explained by anthropometric measures of pelvic geometry, and hamstring and lumbar flexibility.

\section{METHODS}

Participants. Twenty-six experienced cyclists (12 males and 14 females) were recruited via flyers and posters from local cycling groups (Table 1). All subjects were at least $18 \mathrm{yr}$ of age, had been road bicycling for at least $1 \mathrm{yr}$, and were regularly riding at least $3 \mathrm{~h} \cdot \mathrm{wk}^{-1} /$ week at the time of the study. Subjects had no history of saddle sores, skin irritations in the perineal area, perineal nodules, or perineal numbness. Participants gave informed consent in accordance with a protocol approved by the University of Wisconsin's health sciences institutional review board.

Procedures and instrumentation. An adjustable stationary bicycle (Fig. 1) was set up for each subject, using a standardized bicycle-fitting procedure for road racing cyclists (Road Racing Professional Bike Fit, bikefitting.com, the Netherlands). The stationary bicycle was fitted with a gender-neutral saddle (Bontrager X Lite Pro 2006) and standard drops handlebars (400-mm width, $145-\mathrm{mm}$ drop) that were used for all subjects. In addition, a subset of subjects (11 females, 4 males) rode two additional saddles (fi'zi:k Vitesse 2006, Bontrager Race Lite Mens 2006) to assess the influence of saddle factors on pelvic motion. The Vitesse is a women-specific design that has greater compliance in the perineal region and is wider than the X Lite Pro. The Race Lite Mens has both greater compliance and rear curvature than the X Lite Pro. Each saddle was mounted horizontally, using a level aligned along the centerline from the back to the front of the saddle. Care was taken such that dips in the shape of the saddle did

TABLE 1. Subject characteristics and anthropometric measures for the experienced male and female cyclists tested in this study.

\begin{tabular}{lcc}
\hline & Males (mean (SD)) & Females (mean (SD)) \\
\hline$N$ & 12 & 14 \\
Height $(\mathrm{m})$ & $1.81(0.07)$ & $1.69(0.06)^{\star}$ \\
Age $(\mathrm{yr})$ & $36.8(11.1)$ & $34.1(11.2)$ \\
Mass $(\mathrm{kg})$ & $77.1(6.0)$ & $61.2(6.4)^{\star}$ \\
Hip joint center width $(\mathrm{mm})$ & $198.8(12.5)$ & $192.9(9.4)$ \\
Ischial tuberosity width $(\mathrm{mm})$ & $116.5(16.0)$ & $134.9(9.2)^{\star}$ \\
Hamstring flexibility $\left(^{\circ}\right)$ & $31.7(11.8)$ & $27.2(6.5)$ \\
Lumbar flexibility $(\mathrm{cm})$ & $21.2(1.0)$ & $20.6(1.0)$ \\
\hline
\end{tabular}

Although the females were smaller in terms of mass and height, they did exhibit a significantly greater ischial tuberosity width than the males $\left({ }^{*} P<0.05\right)$.

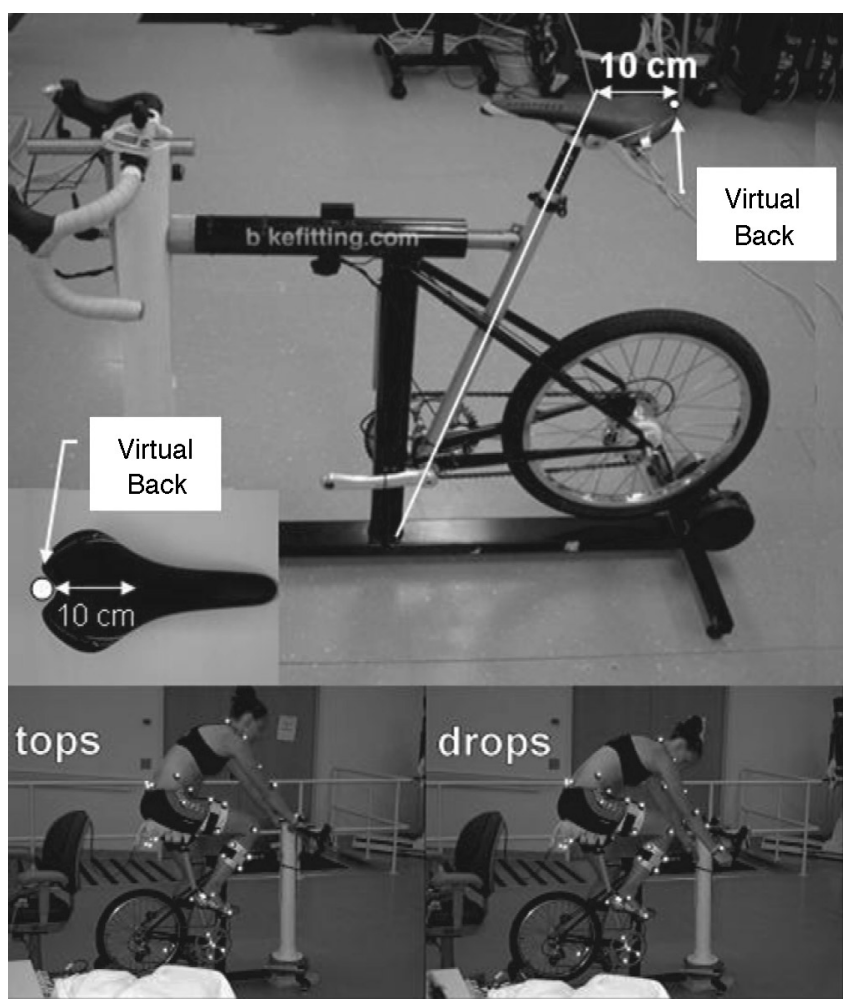

FIGURE 1-An adjustable bicycle was set up for each subject, using a bicycle-fitting procedure based on subject-specific measures of anthropometry. For each subject, the back of the saddle was positioned $10 \mathrm{~cm}$ behind the seat tube, and the saddle was leveled to the horizontal. Subjects pedaled the stationary bicycle at power outputs of 100,150 , and $200 \mathrm{~W}$ with their hands positioned on the tops and drops of the handlebars. Kinematics were recorded using a passive motioncapture system.

not affect the leveling procedure. The posterior aspect of the saddle was positioned $10 \mathrm{~cm}$ from the seat post (Fig. 1). Individualized rider measurements included height, weight, torso height, arm length, inseam length, foot length, shoulder width, bicycle shoe brand, bicycle shoe model, pedal brand, and pedal model. These measures were used to adjust the seat height, handlebar reach, and handlebar height (Fig. 1). After the initial fitting, the bicycle set-up was not changed throughout the duration of the test.

Body segment kinematics were measured at $100 \mathrm{~Hz}$, using an eight-camera motion-capture system (Eagle Digital System; Motion Analysis Corp., Santa Rosa, CA) to track 43 reflective markers placed on the subject. Commercial software (EVaRT v. 4.7; Motion Analysis Corp., Santa Rosa, CA) was used to calibrate the capture space and compute the three-dimensional marker kinematics. Three anatomical markers placed over the second sacral vertebra (S2) and the right and left posterior superior iliac spine (PSIS) were used to track pelvic motion during the pedaling trials. The other markers were used to track upper-body, upper-extremity, and lower-extremity motion. Four markers placed on the bicycle crank were used to monitor the crank angle during cycling. An additional 10 anatomical markers, including two markers on the right and left anterior superior 
iliac spine (ASIS), were used in an upright standing posture to establish segment coordinate systems (Fig. 2). Two hip joint center calibration trials were performed in which the subject circumducted his/her right and left limbs to establish hip center locations in the pelvis reference frame (17).

Each subject warmed up for $5 \mathrm{~min}$ at a self-selected cadence and trainer resistance before data collections. They then performed a series of trials in which power $(100,150$, and $200 \mathrm{~W}$ ) and hand position (tops and drops position) were systematically varied. Cadence was maintained at a constant $90 \mathrm{rpm}$ for all trials. An instrumented rear hub (PowerTap, Saris Corp., Madison, WI) provided the subjects with real-time feedback of their cadence and power output. After the subject achieved a steady power output in a specified posture (1-3 min), marker kinematics were recorded for a minimum of 15 pedal strokes $(10 \mathrm{~s})$.

Data analysis. The processing of the three-dimensional marker kinematics was analyzed for 12 pedal strokes for each trial, which was found to be of sufficient duration to assess a subject's average pelvic motion over a pedal stroke. Kinematic data was first low-pass filtered at $5 \mathrm{~Hz}$, using a quintic smoothing spline (23). The pelvis position and orientation were then computed at each time step, using the

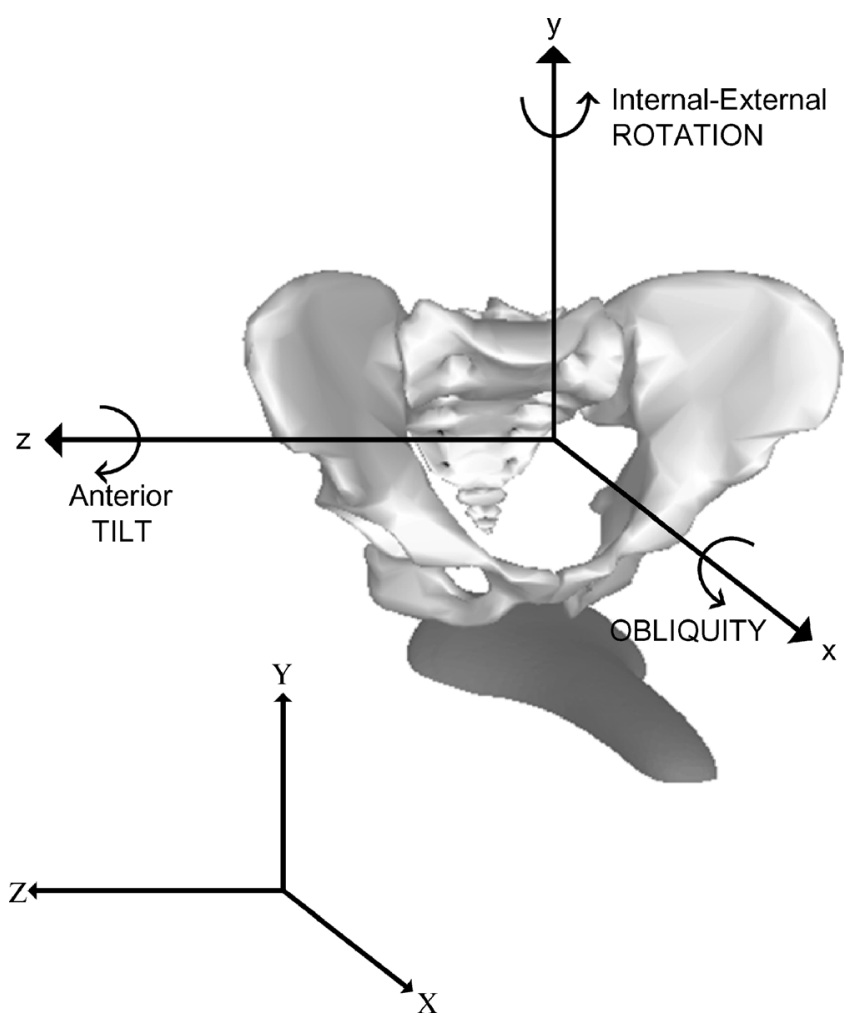

FIGURE 2-The pelvic reference frame, defined by mutually perpendicular $x, y$, and $z$-unit vectors, was centered between the anterior superior iliac spines (ASIS). The $y$-axis direction was defined as perpendicular to a plane defined by the ASIS and posterior anterior iliac spine (PSIS). The translation and orientation of the pelvic reference frame relative to a ground-fixed reference frame (defined by the $x$-, $y$-, and $z$-axes), was used to describe pelvic motion. Body fixed $z-x-y$ (tilt-obliquity-internal/external) rotation angles were used to define the orientation of the pelvis relative to ground. measured positions of the two PSIS markers and the sacral marker (11). Three-dimensional pelvic orientation was defined by a body-fixed $z-x-y$ (anterior-posterior $(A-P)$ tilting followed by obliquity, followed by internal-external $(I-E)$ rotation) rotation sequence (Fig. 2) (4). Right hip motion was determined using the measured pelvic position and orientation, together with the subject-specific location of the hip joint centers relative to the pelvic markers reference frame. SIMM Pipeline (Musculographics Inc., Motion Analysis Corpl, Santa Rosa, CA) was used in conjunction with SD/FAST (Parametric Technology Corporation, Waltham, MA) and custom code to perform the kinematic analyses.

From each pedal stroke, the average, peak, and excursions of the pelvic rotation angles and right hip joint center translations were extracted. Excursion was defined as the range of variation of the rotation angles and hip translations over a single pedal stroke. Kinematic measures were then averaged for the 12 successive pedal strokes from each trial and were used to test the main hypotheses of this study.

Pelvic geometry and flexibility. Pelvic geometry was characterized by the hip joint center-to-center width and the distance between the ischial tuberosities. The latter distance was measured using subject-specific impressions of floral foam blocks. Foam blocks were placed on a foot stool positioned next to a wall. Subjects were instructed to place their back flat against the wall and lower themselves onto the foam. Once an initial impression was felt, the subject was asked to pull up on the base board to ensure that a deep, measurable impression had been made. Ball bearings were then rolled on the foam indentations until they came to rest at the deepest point of the impressions. The distance between the ischial tuberosities was determined by measuring the distance between the ball bearings with a straight edge ruler.

Both hamstring and lumbar flexibility were also assessed for each subject after they had performed the initial pedaling warm-up period. Hamstring flexibility was determined using the standard "90-90" test (16), in which the hip is flexed to $90^{\circ}$ and the knee is then extended until resistance is felt. The knee flexion angle at the end range of motion is used as a measure of hamstring flexibility. Lumbar flexibility was measured using the modifiedmodified-Schober test (16), in which the subject is asked to stand upright with their feet one foot-width apart, and then bend over until resistance is felt. Two marks are made in the standing posture: one on the midpoint between the two PSIS and another $15 \mathrm{~cm}$ superior from this point. The distance between these points is measured again in the bent posture, and this value indicates lumbar flexibility. Each flexibility measure was evaluated three times by the same experimenter, and trials were averaged. Hamstring flexibility was assessed by averaging the knee angle measured on the right and left limbs.

Statistical analysis. The independent variables in this study were gender (male and female), power (100, 150, and 200), and hand position (tops and drops). The dependent 


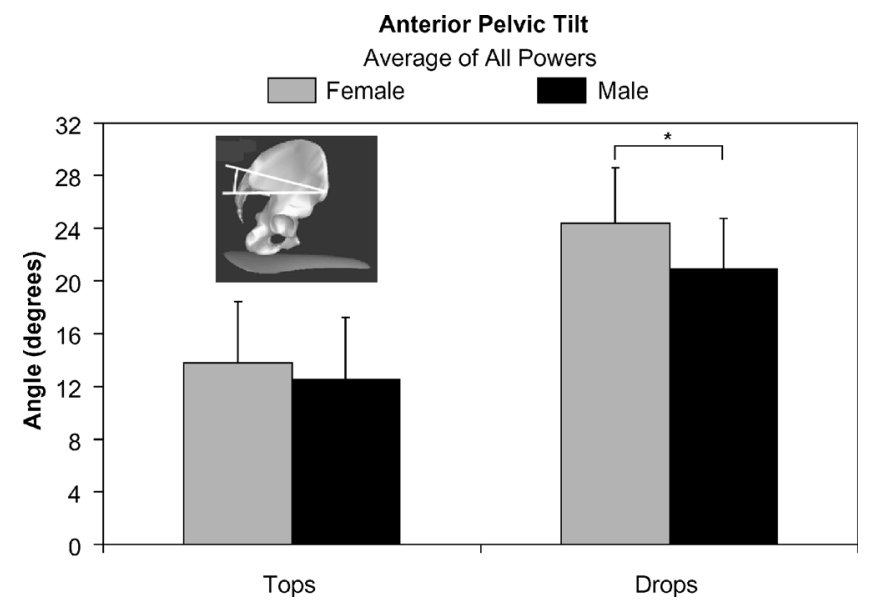

FIGURE 3-The average anterior pelvic tilt was not significantly different between males or females in the tops hand position. However, in the drops hand position, average pelvic tilt was greater for females than for males $(* P=0.036)$. Because power did not significantly affect average pelvic tilt, the mean ( \pm 1 SD) value across all power levels is shown for each gender.

variables were average pelvic tilt, angular excursions $(A-P$ tilt, obliquity, and $I-E$ rotation), and hip translation $(A-P$, inferior-superior $(I-S)$ ) excursions. A three-way analysis of variance was examined for main effects and interactions with repeated measure on the power and hand position factors. Post hoc Tukey's test was used to conduct pairwise comparisons of the main effects. The probability associated with type I error was set at 0.05 for all observations. The relationship of pelvic geometry, hamstring flexibility, and lumbar flexibility with pelvic motion measures was assessed using the Pearson product-moment correlation coefficient. Finally, a pairwise $t$-test was used to evaluate differences between excursions and translations on different saddles, as well as the subjects' characteristics between genders (Table 1).

\section{RESULTS}

Anthropometric data. Females exhibited a significantly $(P<0.001)$ larger average distance between the ischial tuberosities than did males $(134.9 \mathrm{~mm}$ vs $116.5 \mathrm{~mm}$, Table 1). There were no significant gender-related differences in hip joint center-to-center width, hamstring flexibility, or lumbar flexibility.

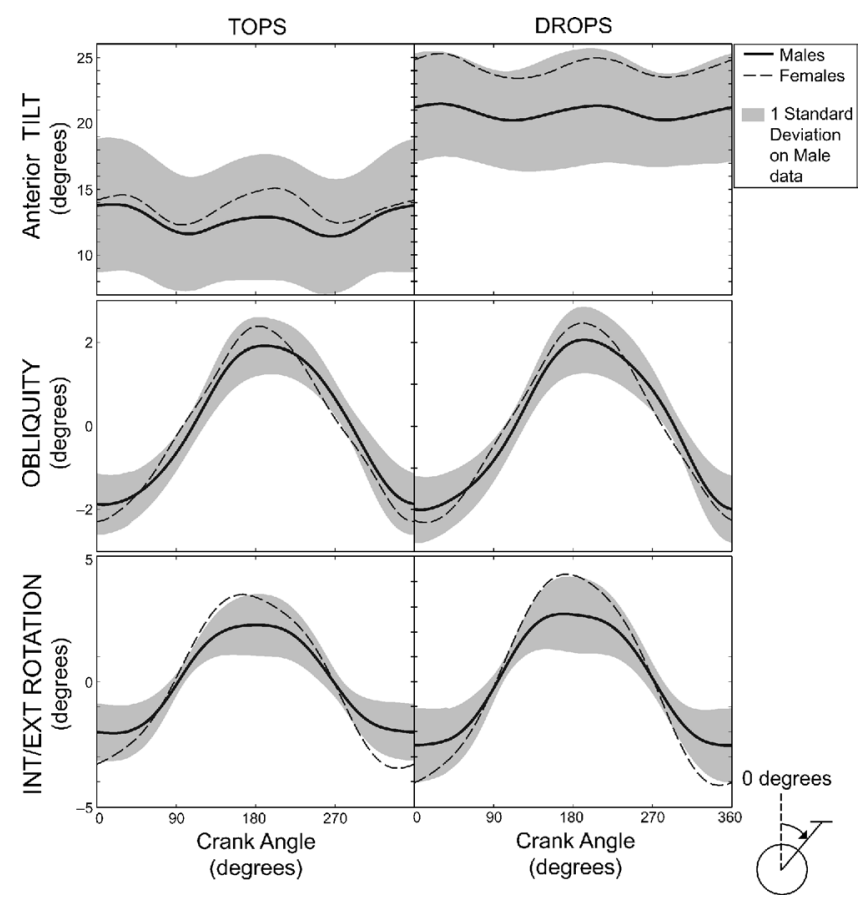

FIGURE 4-Ensemble-average ( \pm 1 SD) pelvic rotation angles throughout a pedal stroke at $200 \mathrm{~W}$ in the tops and drops hand position. The largest angular excursions of the pelvis were observed in the nonsagittal planes, with the pelvis internally rotating and rolling toward the downstroke on each side. Moving from the tops to the drops position increased the average anterior pelvic tilt but did not substantially alter the shape of the angular excursions. Zero crank angle was defined as the top dead center position.

Pelvic orientation. There was a significant gender and position interaction on anterior pelvic tilt $(P=0.031$, $\left.F_{1,24}=5.27\right)$, with females exhibiting significantly $(P=$ $0.036)$ greater pelvic tilt in the drops hand position compared with males (24.4 vs $20.9^{\circ}$, Fig. 3). This result was observed at all power outputs.

Pelvic motion during a pedal stroke. The average angular excursions across a pelvic pedal stroke ranged from $2^{\circ}$ in anterior pelvic tilt in the tops position, to $8^{\circ}$ in $I-E$ rotation in the drops position (Table 2). $A-P$ tilting of the pelvis oscillated twice a pedal stroke with maximum anterior tilt corresponding to the top dead center position of the crank (Fig. 4). The pelvis rotated internally $\left(\sim 3^{\circ}\right)$ and rolled laterally $\left(\sim 2^{\circ}\right)$ toward the downstroke side, reaching peak internal rotation and obliquity angles near bottom dead

TABLE 2. Mean (SD) angular excursions in pelvic tilt, obliquity, and internal-external rotation across a pedal stroke for both genders at each of the test conditions.

\begin{tabular}{|c|c|c|c|c|c|c|c|}
\hline \multirow[b]{2}{*}{ Posture Hand Position } & \multirow[b]{2}{*}{ Power (W) } & \multicolumn{2}{|c|}{ Tilt Excursion (deg) } & \multicolumn{2}{|c|}{ Obliquity Excursion (deg) } & \multicolumn{2}{|c|}{ Int-Ext Rotation Excursion (deg) } \\
\hline & & $\begin{array}{l}\text { Male, Mean } \\
\text { (SD) }\end{array}$ & $\begin{array}{c}\text { Female, Mean } \\
\text { (SD) }\end{array}$ & $\begin{array}{l}\text { Male, Mean } \\
\text { (SD) }\end{array}$ & $\begin{array}{l}\text { Female, Mean } \\
\text { (SD) }\end{array}$ & $\begin{array}{l}\text { Male, Mean } \\
\text { (SD) }\end{array}$ & $\begin{array}{c}\text { Female, Mean } \\
\text { (SD) }\end{array}$ \\
\hline Tops & 100 & $3.8(1.9)$ & $4.0(1.5)$ & $4.5(1.4)$ & $4.5(2.0)$ & $4.9(2.0)$ & $5.9(1.9)$ \\
\hline Tops & 150 & $3.9(2.0)$ & $4.0(1.4)$ & $4.4(1.3)$ & $4.7(2.3)$ & $4.9(2.0)$ & $6.4(2.2)$ \\
\hline Tops & 200 & $3.8(1.7)$ & $4.1(1.5)$ & $4.2(1.3)$ & $4.1(2.6)$ & $5.1(2.2)$ & $7.5(2.4)$ \\
\hline Drops & 100 & $2.1(1.4)$ & $2.7(1.8)$ & $4.7(1.2)$ & $5.0(2.0)$ & $5.8(2.5)$ & $7.7(2.8)$ \\
\hline Drops & 150 & $2.1(1.6)$ & $2.6(1.6)$ & $4.5(1.2)$ & $5.0(2.0)$ & $5.7(2.3)$ & $8.1(2.9)$ \\
\hline Drops & 200 & $2.4(1.7)$ & $2.6(1.7)$ & $4.5(1.5)$ & $5.2(2.3)$ & $6.1(1.5)$ & $8.8(2.3)$ \\
\hline
\end{tabular}




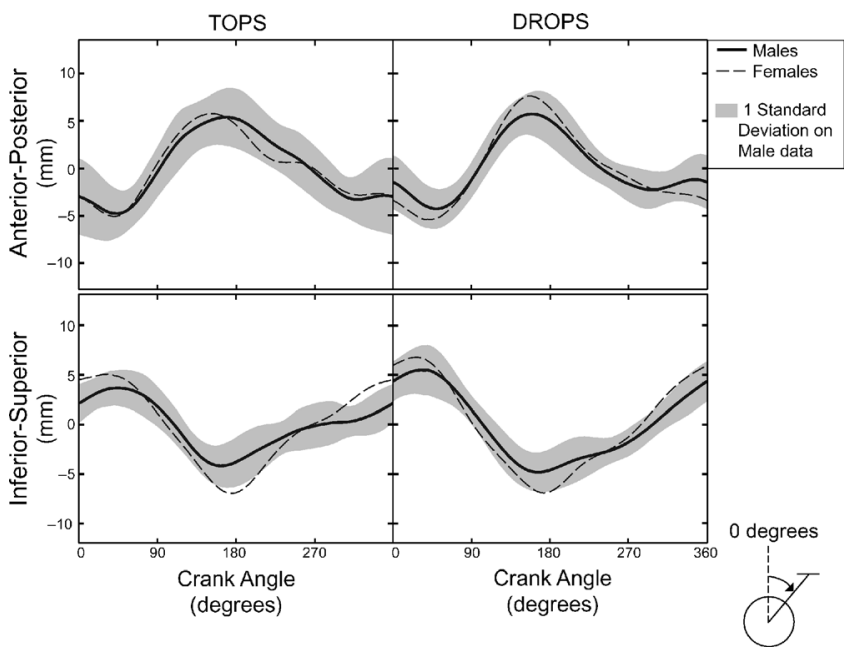

FIGURE 5-Ensemble-average ( \pm 1 SD) right hip joint center motion throughout a pedal stroke at $200 \mathrm{~W}$ in the tops and drops hand position. The hip translated anteriorly and posteriorly during much of the right limb downstroke. Moving from the tops to the drops posture increased the inferior-superior translations but did not substantially alter the characteristic motion. Zero crank angle was defined as the top dead center position.

center. These pelvic rotations caused the hip on the downstroke side to translate anteriorly and inferiorly during much of the downstroke (Fig. 5).

Pelvic and hip motion. There was a significant interaction between power and gender on $I-E$ pelvic rotation $\left(P<0.001, F_{2,48}=10.29\right.$, Table 3$)$. Males did not increase $I-E$ excursions with power $(P=0.627-0.999)$, whereas females exhibited significantly increasing $I-E$ excursions with power $(P<0.001$ from 100 to $200 \mathrm{~W}$ and from 150 to $200 \mathrm{~W}$ ) (Fig. 6). In addition, there was a significant interaction between power and gender on pelvic obliquity $\left(P=0.009, F_{2,48}=5.27\right)$ and $I-S$ hip translation $\left(P=0.040, F_{2,48}=3.45\right)$, with females exhibiting larger motion in both these degrees of freedom as power increased (Fig. 6). Switching from the top to the drops hand position caused a significant decrease in the anterior tilt excursion $\left(P<0.001, F_{1,24}=15.91\right)$, a significant increase in $I-E$ rotation $\left(P<0.001, F_{1,24}=49.00\right)$, and greater vertical hip translation $\left(P=0.002, F_{1,24}=11.29\right)($ Tables $3-5)$. There was also a significant interaction between gender and position in the $A-P$ hip translation $\left(P=0.001, F_{1,24}=\right.$ $12.98)$, with females exhibiting significantly more $A-P$ hip motion in the drops hand position $(P=0.029)$.

Correlation with anthropometry and flexibility. There were no significant correlations between ischial tuberosity width, hip joint center-to-center distance, or hamstring flexibility with the average pelvic tilt or angular excursions. Among the males, the average anterior pelvic
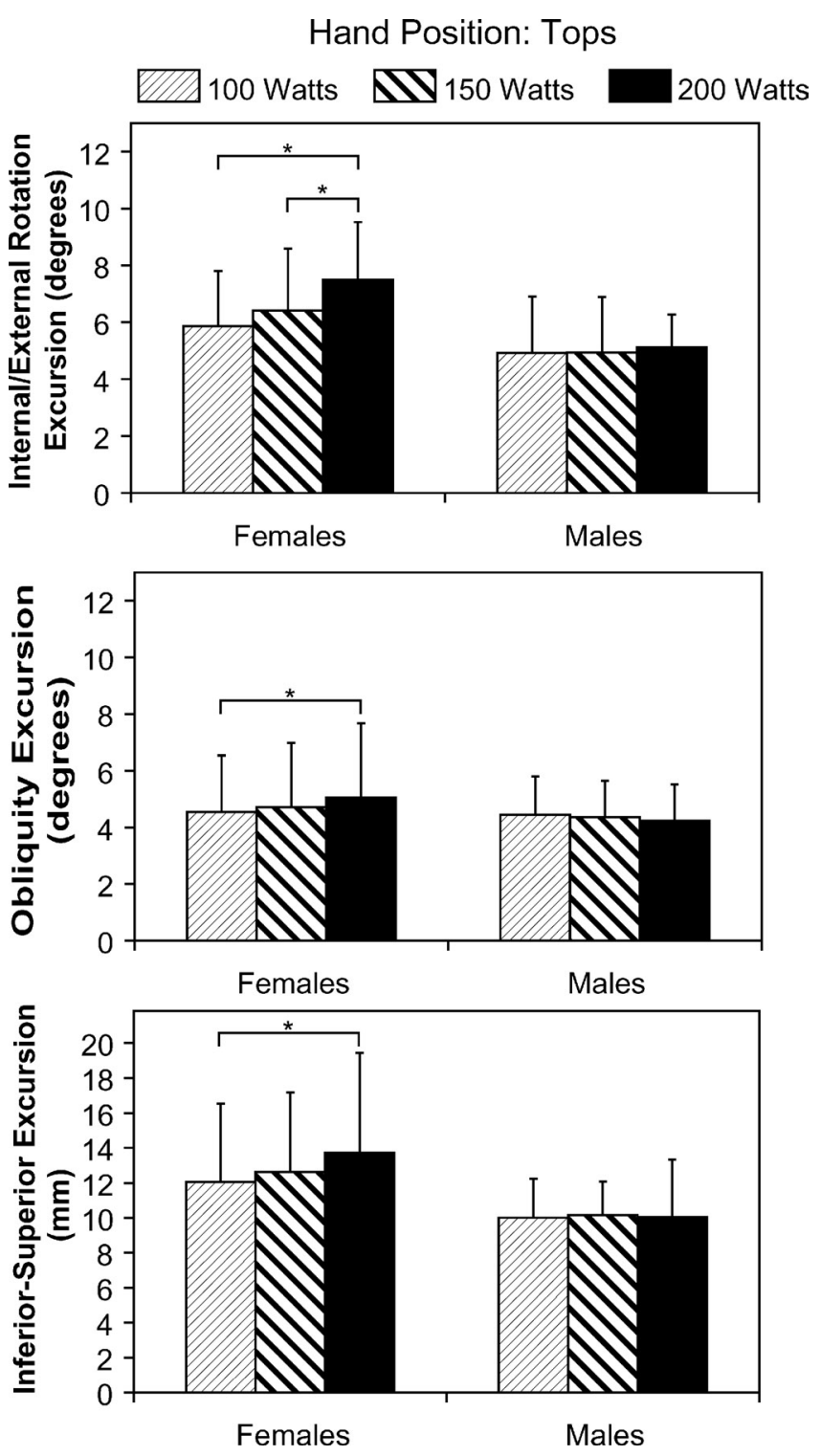

FIGURE 6-The effect of power on pelvic motion in the top hand position. The excursion of internal-external rotation increased with power output for the females, but it was independent of power output for the males. Power-related differences $(* P<0.05)$ in pelvic obliquity excursions and vertical hip translations were also evident in the females between the low $(100 \mathrm{~W})$ and high $(200 \mathrm{~W})$ power output conditions. Similar results were obtained in the drops hand position.

tilt in the drops hand position was negatively correlated $(r=0.733-0.767 ; P<0.05)$ with lumbar flexibility at all powers (Fig. 7).

Pelvic motion on other saddles. The characteristic patterns of pelvic motion were similar across all three test saddles for the subset $(N=14)$ of subjects who rode them all in the tops and drops hand position at $200 \mathrm{~W}$. Under

TABLE 3. The effects of gender, power, and hand position on the angular excursions in pelvic tilt, obliquity, and internal-external rotation (ANOVA, * $P<0.05)$.

\begin{tabular}{|c|c|c|c|c|c|c|c|}
\hline Measure & Gender & Power & Position & Gender*Power & Gender*Position & Power*Position & Gender ${ }^{\star}$ Power ${ }^{\star}$ Position \\
\hline Pelvic tilt & 0.519 & 0.347 & $<0.001^{*}$ & 0.941 & 0.75 & 0.833 & 0.318 \\
\hline Obliquity & 0.536 & 0.644 & 0.092 & $0.009^{*}$ & 0.839 & 0.187 & 0.328 \\
\hline Int-ext rotation & $0.039 *$ & $<0.001^{*}$ & $<0.001^{*}$ & $<0.001^{*}$ & 0.057 & 0.580 & 0.342 \\
\hline
\end{tabular}


TABLE 4. Mean (SD) excursions of hip joint center translation in the anterior-posterior $(x)$ direction and inferior-superior $(y)$ direction across a pedal stroke for both genders at each of the test conditions.

\begin{tabular}{|c|c|c|c|c|c|}
\hline \multirow[b]{2}{*}{ Posture Hand Position } & \multirow[b]{2}{*}{ Power (W) } & \multicolumn{2}{|c|}{ Anterior-Posterior Excursion (mm) } & \multicolumn{2}{|c|}{ Inferior-Superior Excursion (mm) } \\
\hline & & Male, Mean (SD) & Female, Mean (SD) & Male, Mean (SD) & Female, Mean (SD) \\
\hline Tops & 100 & $14.1(3.4)$ & $12.1(3.9)$ & $10.0(2.3)$ & $12.0(4.5)$ \\
\hline Tops & 150 & $13.8(3.5)$ & $11.6(4.3)$ & $10.2(1.9)$ & $12.6(4.6)$ \\
\hline Tops & 200 & $14.2(4.0)$ & $12.9(4.6)$ & $10.0(2.4)$ & $13.7(5.2)$ \\
\hline Drops & 100 & $12.6(3.8)$ & $14.0(4.3)$ & $12.1(3.3)$ & $14.0(5.7)$ \\
\hline Drops & 150 & $12.0(3.3)$ & $14.1(4.5)$ & $11.9(3.4)$ & $14.1(5.4)$ \\
\hline Drops & 200 & $12.6(4.0)$ & $14.8(4.5)$ & $11.8(3.8)$ & $14.7(4.9)$ \\
\hline
\end{tabular}

these test conditions, the saddle tested had no significant effect on the average anterior pelvic tilt, vertical hip translation, or the angular excursions in the sagittal or frontal planes. There were, however, slightly greater $I-E$ angular excursions in the top hand position for the Vitesse $(3.7 \pm$ $\left.1.6^{\circ} ; P=0.010\right)$ and Race Lite Mens $\left(3.4 \pm 1.5^{\circ} ; P=0.018\right)$ when compared with the X Lite Pro $\left(3.1 \pm 1.3^{\circ}\right)$. In the drops hand position, riders exhibited slightly greater $I-E$ rotational excursions on the Vitesse $\left(4.2 \pm 1.7^{\circ} ; P=0.012\right)$ had slightly greater $I-E$ excursions than did the $\mathrm{X}$ Lite Pro $\left(3.7 \pm 1.4^{\circ}\right)$.

\section{DISCUSSION}

This study demonstrates that substantial three-dimensional pelvic motion occurs naturally among both experienced male and female cyclists during seated cycling. The largest angular excursions were observed in the nonsagittal planes, causing the hip to translate forward and down during the downstroke of cycling. This hip motion would contribute to a transfer of energy from the upper to lower body via the hip joint reaction force (14). The sagittal hip motion was relatively independent of power output, except for a slight increase in vertical translation among females in the tops position. Thus, the power transfer from the upper to lower body would increase with power output because of an increase in hip reaction force with power output, a result that would be consistent with that found by others $(6,14,22)$.

Gender-related differences in pelvic orientation emerged when changing hand position, with the females exhibiting greater anterior tilt $\left(\sim 3^{\circ}\right.$ more $)$ in the drops hand position, when compared with the males. The absolute magnitude of pelvic tilt angles are about $3-4^{\circ}$ less than the angles reported by Bressel et al. (3), but they are comparable with the pelvic tilt measured by Mueller et al. (13). Differences between studies may be attributable to differing measuring techniques. Bessel et al. (3) used an inclinometer to manually measure pelvic tilt in a static posture, whereas the current study used reflective markers placed on the PSIS and S2 joints to measure full 3D orientation during pedaling. Though the current study did not find significant differences in lumbar flexibility between genders, lumbar flexibility was negatively correlated with pelvic tilt among the males, suggesting this was a relevant factor affecting pelvic orientation. It is also possible that the gender-related differences in pelvic tilt could arise, in part, from the bicycle set-up that was used. In particular, the same handlebars were used for all testing, meaning that the vertical change in the hand position from the tops to the drops $(145 \mathrm{~mm})$ was the same for all subjects, which may have required the females to rotate further forward when moving to the drops hand position.

The females exhibited increased excursions in pelvic obliquity and internal rotation with increasing power output, a trend not observed among the males. The difference could reflect an alternative pedaling strategy or anatomical differences. All cyclists pedaled at the same absolute powers in this study, which placed the females at a larger percentage of their maximum power output. Thus, there is the possibility that the larger pelvic motion occurred only when approaching maximum power output and that males would have shown similar trends if they had further increased their power output. If, instead, the differences were attributable to anatomical factors, these did not seem to be captured by simple measures of pelvic geometry, lumbar flexibility, and hamstring flexibility, because none of these factors correlated significantly with interindividual differences in pelvic angular excursions.

Ischial tuberosity width could not independently explain interindividual differences in pelvic orientation, angular excursions, or hip motion. The lack of a correlation may arise from the riding posture assumed by the road cyclists. Whereas upright cyclists may support their weight on the ischial tuberosities $(10,19,20)$, the road cyclists in this study tilted their pelves to such a degree that they were more supported on their ischiopubic rami and perhaps also on the pubis in the drops position (12). Thus, although pelvic geometry may play a role in the pelvic position assumed on the saddle, this geometry may not be sufficiently described by the ischial tuberosity width. Other factors such as the rami angle, pubic arch, and soft tissues may also need to be considered in determining the structures providing support (1).

TABLE 5. The effects of gender, power, and hand position on the excursions in hip joint center translation across a pedal stroke (ANOVA, $\left.{ }^{*} P<0.05\right)$.

\begin{tabular}{lccccccc}
\hline Measure & Gender & Power & Position & Gender ${ }^{*}$ Power & Gender ${ }^{*}$ Position & Power*Position & Gender Power*Position \\
\hline$A-P$ excursion & 0.971 & 0.058 & 0.633 & 0.517 & $\mathbf{0 . 0 0 1}^{*}$ & 0.844 \\
I-S excursion & 0.125 & 0.116 & $\mathbf{0 . 0 0 3 ^ { * }}$ & $\mathbf{0 . 0 3 4 ^ { * }}$ & 0.683 & 0.145 \\
\hline
\end{tabular}




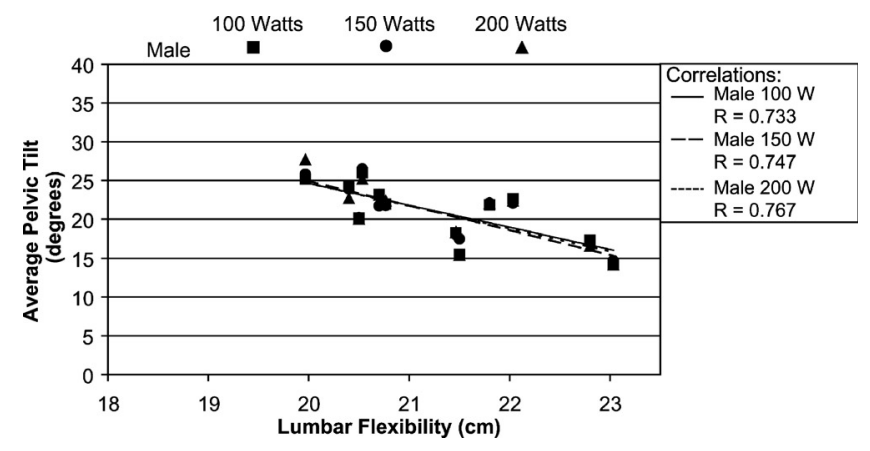

FIGURE 7-Males exhibited a significant negative correlation $(P<$ 0.05 ) between average anterior pelvic tilt and lumbar flexibility. This effect was not observed among the females. The drops hand position is shown, though similar results were observed in the tops hand position.

A limitation of the current study was the use of skinbased markers to characterize pelvic motion, which are subject to error attributable to soft-tissue motion. In addition, the absolute resolution of our angular excursions though could be slightly limited by the use of a triad of markers in close spatial proximity (Fig. 1). The average distance between the most inferior and superior markers in the sagittal plane was $16 \mathrm{~mm}$, whereas the average horizontal distance between the most lateral markers was $97 \mathrm{~mm}$. This would suggest that marker kinematic errors would corrupt anterior tilt angles more than obliquity angles. However, the magnitude of our estimated hip motion was similar to the results reported by Neptune and Hull (14) using bone pins, and it was substantially smaller than estimates obtained using a single marker located over the greater trochanter $(7,22)$ or ASIS (14). This would suggest that our approach of using a triad of posterior pelvic markers to track pelvic motion can provide reasonable estimates of hip motion in experienced road cyclists. Because the hip motion arises, in part, from the pelvic rotation, this also increases our confidence in using skinbased markers to estimate pelvic rotations.

The results obtained in this study are relevant for saddle design. In particular, substantial pelvic motion was observed throughout a pedal stroke on all three saddles

\section{REFERENCES}

1. BRESSEL, E. Influence of bicycle seat pressure on compression of the perineum: a MRI analysis. J. Biomech. 40:198-202, 2005.

2. Bressel, E., and J. Cronin. Bicycle seat interface pressure: reliability, validity, and influence of hand position and workload. J. Biomech. 38:1325-1331, 2005.

3. Bressel, E., and B. J. Larson. Bicycle seat designs and their effect on pelvic angle, trunk angle, and comfort. Med. Sci. Sports Exerc. 35:327-332, 2003 .

4. Cole, G. K., B. M. NigG, J. L. Ronsky, and M. R. Yeadon. Application of the joint coordinate system to three-dimensional joint attitude and movement representation: a standardization proposal. J. Biomech. Eng. 115:344-349, 1993.

5. Fregly, B. J., F. E. Zajac, and C. A. Dairaghi. Crank inertial load has little effect on steady-state pedaling coordination. J. Biomech. 29:1559-1567, 1996. tested, suggesting that this motion arises naturally during pedaling. Thus, there is a need to design saddles to accommodate such motion without subjecting the rider to undue pressure or shear at the saddle interfaces. The transition region between the back of the saddle and the nose is one area of concern because of the forward and downward motion of the hip during the downstroke, which could contribute to shear loading at this interface. In addition, the rear-view curvature of the saddle should be designed to support the ischiopubic rami while allowing for side-to-side pelvic roll. Furthermore, the large anterior tilt observed means that road cyclists are partially supported on the anterior pelvic structures. This puts increased emphasis on designing the saddle contour to support the ischiopubic rami while not excessively stressing tissue in the perineum and pubic arch, the loading of which has been linked to saddle pathologies such as a painful pubic area, chaffing, saddle sores, pudendal neuropathy, genital numbness, and erectile dysfunction $(1,8,9,19)$.

This study also provides information relevant for fitting a bicycle to minimize discomfort and risk for saddle-related pathologies. Although observable pelvic rocking can be indicative of too high a saddle position (12), this study demonstrates that some pelvic roll arises naturally during pedaling. Thus, the goal of setting the saddle height in bicycle fitting should not necessarily be to completely eliminate pelvic roll, because the pelvic motion likely serves to facilitate power transfer between the upper and lower body (14). Furthermore, the link between pelvic tilt and lumbar flexibility highlights the need to consider an individual's range of motion in bicycle fitting.

In summary, we observed substantial pelvic motion during seated cycling with experienced female road cyclists exhibiting greater anterior tilt and nonsagittal pelvic rotations in a drops hand position than their male counterparts. This information, along with systematic evaluation of saddle pressure distributions, could prove useful for scientifically establishing guidelines for saddle design and bicycle fitting.

We gratefully acknowledge the contributions of Matt Prest and Damon Rinard, and the support provided by Trek Bicycle Corp.

6. Gregor, R. J., J. P. Broker, and M. M. Ryan. The biomechanics of cycling. Exerc. Sport Sci. Rev. 19:127-169, 1991.

7. Gregor, R. J., P. R. Cavanagh, and M. LaFortune. Knee flexor moments during propulsion in cycling-a creative solution to Lombard's paradox. J. Biomech. 18:307-316, 1985.

8. Guess, M. K., K. Connell, S. Schrader, et al. Genital sensation and sexual function in women bicyclists and runners: are your feet safer than your seat? J. Sex Med. 3:1018-1027, 2006.

9. Keytel, L. R., and T. D. Noakes. Effects of a novel bicycle saddle on symptoms and comfort in cyclists. S. Afr. Med. J. 92:295-298, 2002.

10. Lowe, B. D., S. M. Schrader, and M. J. Breitenstein. Effect of bicycle saddle designs on the pressure to the perineum of the bicyclist. Med. Sci. Sports Exerc. 36:1055-1062, 2004. 
11. Lu, T. W., and J. J. O'Connor. Bone position estimation from skin marker co-ordinates using global optimisation with joint constraints. J. Biomech. 32:129-134, 1999.

12. Mestdagh, K. d. V. Personal perspective: in search of an optimum cycling posture. Appl. Ergon. 29:325-334, 1998.

13. Mueller, P., M. McEvoy, and S. Everett. The long sitting screening test in elite cyclists. J. Sci. Med. Sport 8:369-374, 2005.

14. Neptune, R. R., and M. L. Hull. Accuracy assessment of methods for determining hip movement in seated cycling. J. Biomech. 28:423-437, 1995.

15. Neptune, R. R., and A. J. van den Bogert. Standard mechanical energy analyses do not correlate with muscle work in cycling. $J$. Biomech. 31:239-245, 1998

16. Norkin, C. C., and J. White. Measurement of Joint Motion: A Guide to Goniometry, 3rd ed. Philadelphia, PA: F.A. Davis Company, 2003.

17. Piazza, S. J., A. Erdemir, N. Okita, and P. R. Cavanagh. Assessment of the functional method of hip joint center location subject to reduced range of hip motion. J. Biomech. 37:349-356, 2004.
18. Rodano, R., R. Squadrone, M. Sacchi, and A. Marzegan. Saddle pressure distribution in cycling: comparison among saddles of different design and materials. In: International Symposium on Biomechanics in Sports. Cáceres, Spain: ISBS, pp. 606-609, 2002.

19. Silberman, M. R., D. Webner, S. Collina, and B. J. Shiple. Road Bicycle Fit. Clin. J. Sport Med. 15:271-276, 2005.

20. Spears, I. R., N. K. Cummins, Z. Brenchley, et al. The effect of saddle design on stresses in the perineum during cycling. Med. Sci. Sports Exerc. 35:1620-1625, 2003.

21. Stone, C., and M. L. Hull. The effect of rider weight on riderinduced loads during common cycling situations. J. Biomech. 28:365-375, 1995.

22. van Ingen Schenau, G. J., W. W. van Woensel, P. J. Boots, R. W. SNACKERS, and G. DE GROOT. Determination and interpretation of mechanical power in human movement: application to ergometer cycling. Eur. J. Appl. Physiol. Occup. Physiol. 61:11-19, 1990.

23. Woltring, H. J. A FORTRAN package for generalized crossvalidation spline smoothing and differentiation. Adv. Engng. Softw. 8:104-108, 1986. 